\title{
HEMOSTATIC POTENTIAL ASSESSMENT OF PATIENTS WITH LIVER CIRRHOSIS AND ATRIAL FIBRILLATION BY LOW-FREQUENCY PIEZOELECTRIC THROMBOELASTOGRAPHY
}

DOI: $10.36740 /$ WLek202103203

\author{
Alina E. Baylo ${ }^{1}$, Tetiana 0. Maksymets' ${ }^{2}$, Vadym P. Shypulin'1, Volodymyr V. Chernyavskyi' ${ }^{1}$ Luiza M. Parunian' \\ 'BOGOMOLETS NATIONAL MEDICAL UNIVERSITY, KYIV, UKRAINE \\ ¿«MOTHER AND CHILD» MEDICAL CENTER, KYIV, UKRAINE
}

\begin{abstract}
The aim: Our aim was to assess the hemostatic potential of patients with liver cirrhosis and atrial fibrillation by LPTEG global coagulation assay, to investigate changes in LPTEG parameters according to the stage of liver cirrhosis and compare results with liver cirrhosis group.

Materials and methods: We performed a prospective cross-sectional study including 70 patients with liver cirrhosis and atrial fibrillation, 36 patients with liver cirrhosis and 20 healthy individuals. LPTEG parameters were measured using ARP-01M “Mednord" in order to assess coagulation abnormalities.

Results: $\mathrm{t} 1$ and Intensity of contact coagulation didn't differ ( $p>0,05)$, Constant of thrombin activity was increased (47.53 $\pm 0.8 v s .34 .51 \pm 1.88, p<0.001)$, $t 3$ was reduced $(5,0 \pm 0.1 v s .6 .7 \pm 0.36 p<0.001)$, Intensity of coagulation drive was increased ( $52.8 \pm 1.8 v s .38 .55 \pm 1.54, p=0.001)$, Intensity of clot polymerization was increased (19.66 $\pm 0.28 v s .16 .29 \pm 0.28$, $\mathrm{p}<0.001)$, time 5 was reduced ( $32.94 \pm 0.36 \mathrm{vs.} 36.8 \pm 1.30, \mathrm{p}<0.01)$, Maximum amplitude was increased (655.7 $\pm 9.19 \mathrm{vs.} 547 \pm 19.38, \mathrm{p}<0.001)$, Intensity of total coagulation was increased (19.41 $\pm 0.34 v s .15,09 \pm 0.56, p<0.001)$, Intensity of clot retraction and lysis was increased $(4.1 \pm 0.07 v s .3 \pm 0.15, p<0.001)$ and Coefficient of total anticoagulant activity was increased $(2.81 \pm 0.05$ vs. $2.48 \pm 0.06, p<0.001)$ compared to liver cirrhosis.

Conclusions: In patients with liver cirrhosis and atrial fibrillation the hemostatic potential is significantly shifted towards hypercoagulation with a gradual worsening of coagulation disorders, starting from the compensated stage of liver cirrhosis.
\end{abstract}

KEY WORDS: liver cirrhosis, atrial fibrillation, LPTEG, hypercoagulation

Wiad Lek. 2021;74(3 p.II):578-583

\section{INTRODUCTION}

Liver cirrhosis and atrial fibrillation are advanced chronic diseases that occupy a leading position in the structure of mortality and morbidity in the world. [1,2] According to the WHO, the prevalence of liver cirrhosis in the world is approximately $4.5 \%-9.5 \%$ of the total population and increases every year, despite the improvement and spread of vaccination programs, use of new methods of diagnosis and treatment of chronic liver diseases. [3] The course, complications and mortality rate due to liver cirrhosis are significantly influenced by concomitant diseases of the cardiovascular system, among which one of the most important is atrial fibrillation (AF). AF occurs in $1-2 \%$ (33.5 million) of the population and is one of the most common cardiac arrhythmias in the world, affecting mostly the elderly. [4,5] According to statistical data, AF is observed in 5\% (95\% CI:2.8-8.6) patients with liver cirrhosis, which is higher than the prevalence of AF in the general population and is accompanied by a higher levels of hospitalization, mortality and more frequent occurrence of ischemic stroke and acute renal failure compared with liver cirrhosis. [6,7]

Traditionally patients with liver cirrhosis were thought to be "auto-anticoagulated" and prone to bleeding. This theory was supported by changes in standard coagulation parameters, par- ticularly the international normalized ratio(INR), prothrombin time (PT), and clinically - by frequent episodes of bleeding. However, during the last 15 years, more detailed data have emerged that more accurately explain the hemostasis process in chronic liver disease. For a long time, it was not taken into account that along with procoagulant factors the synthesis of physiologic anticoagulants such as antithrombin III, protein $\mathrm{S}$ and $\mathrm{C}$ are reduced, which leads the hemostasis to a new rebalanced state. $[8,9,10]$ The balance of the system may shift towards thrombosis or bleeding depending on various clinical factors - infection, systemic inflammation, renal failure. [11] Atrial fibrillation is a well-known clinical factor that increases the level of thrombotic complications and can theoretically shift the state of hemostasis in patients with liver cirrhosis towards hypercoagulation, provoking various thrombotic complications. [12]

The common laboratory coagulation parameters available in practice focus on the assessment of isolated stages of hemostasis, mainly procoagulant factors, and do not take into account the compensatory mechanisms that balance this process. These tests are performed on plasma, not the whole blood, and thus do not reflect vWF levels, platelet function and other factors that contribute to blood clot formation. Because these methods do not allow to assess the complex 
Table I. Clinical characteristics of patients with liver cirrhosis and AF, liver cirrhosis and healthy donors, number (\%) or mean \pm SD.

\begin{tabular}{|c|c|c|c|}
\hline Parameters & Liver cirrhosis and AF & Liver cirrhosis & Healthy donors \\
\hline Age, years & $68,3 \pm 1,08$ & $58,3 \pm 1,45$ & $61,4 \pm 2,35$ \\
\hline \multicolumn{4}{|l|}{ Sex: } \\
\hline Male & $46(65,71 \%)$ & $22(61,11 \%)$ & $14(70 \%)$ \\
\hline Female & $24(34,29 \%)$ & $14(38,89 \%)$ & $6(30 \%)$ \\
\hline \multicolumn{4}{|l|}{ Etiology: } \\
\hline Alcohol & $28(40 \%)$ & $16(44,4 \%)$ & n.a \\
\hline HCV & $4(5,71 \%)$ & $5(13,89 \%)$ & n.a \\
\hline HBV & $7(10,01 \%)$ & $4(11,12 \%)$ & n.a \\
\hline NAFLD & $19(27,16 \%)$ & $7(19.48 \%)$ & n.a \\
\hline Cardiac cirrhosis & $3(4,26 \%)$ & n.a & n.a \\
\hline Combined & $9(12,86 \%)$ & $4(11,1 \%)$ & n.a \\
\hline \multicolumn{4}{|l|}{ Child-Pugh score: } \\
\hline $\mathrm{A}$ & $25(35,7 \%)$ & $13(36,1 \%)$ & n.a \\
\hline B & $31(44,3 \%)$ & $16(44,4 \%)$ & n.a \\
\hline $\mathrm{C}$ & $14(20 \%)$ & $7(19,45 \%)$ & n.a \\
\hline \multicolumn{4}{|l|}{ Liver cirrhosis duration: } \\
\hline Currently diagnosed & $14(20 \%)$ & n.a & n.a \\
\hline $1-5$ years & $23(32,86 \%)$ & $20(55,6 \%)$ & n.a \\
\hline $6-10$ years & $28(40 \%)$ & $14(38,9 \%)$ & n.a \\
\hline$>11$ years & $5(7,14 \%)$ & $2(5,5 \%)$ & n.a \\
\hline \multicolumn{4}{|l|}{ Antiagregants use: } \\
\hline Clopidogrel & $2(2,86 \%)$ & $2(5,56 \%)$ & n.a \\
\hline Aspirin & $15(21,43 \%)$ & $8(22,2 \%)$ & n.a \\
\hline \multicolumn{4}{|l|}{ Anticoagulants use: } \\
\hline Warfarin & $17(24,3 \%)$ & n.a & n.a \\
\hline Dabigatran & n.a & n.a & n.a \\
\hline Rivaroxaban & $4(5,71 \%)$ & n.a & n.a \\
\hline Apixaban & $1(1,43 \%)$ & n.a & n.a \\
\hline \multicolumn{4}{|l|}{ History of thrombotic events: } \\
\hline Thromboembolic (MI, IS) & $7(10 \%)$ & $1(2,78 \%)$ & n.a \\
\hline Splanchnic vein thrombosis & $9(12,85 \%)$ & $3(8,34 \%)$ & n.a \\
\hline \multicolumn{4}{|l|}{ History of hemorrhagic events: } \\
\hline Gl bleeding & $6(8,57 \%)$ & $3(8,33 \%)$ & n.a \\
\hline Epistaxis & $5(7,14 \%)$ & $3(8,33 \%)$ & n.a \\
\hline Gingival bleeding & $2(2,86 \%)$ & $1(2,76 \%)$ & n.a \\
\hline
\end{tabular}

* MI-Myocardial infarction, IS - Ischemic stroke.

overall picture of rebalanced hemostasis in patients with liver cirrhosis, global methods of hemostasis are becoming increasingly popular, the main principle of which is to assess the clot strength during formation and dissolution in whole blood. $[13,14,15]$ They provide assessment of whole blood coagulation at a more global and clinically feasible level, as they can be performed quickly, in minutes, and therefore rapidly correct a variety of hemostasis disorders. One of such methods is low-frequency piezoelectric thromboelastography (LPTEG). The advantages of LPTEG compared to other VETs are the abilities to assess aggregation activity of thrombocytes, fibrinolytic system, physiological anticoagulant activity and total hemostatic potential of whole blood at any time during the process and specific sample without use of sodium citrate or other components that may affect the result. Also, LPTEG is a completely standardized test with a general sensitivity of all devices' measurements, validated by the manufacturer, which has a certain algorithm for assessing the hemostatic profile, which simplifies its use in clinical settings. [16] There are currently no data on the effect of AF on hemostasis in patients with liver cirrhosis, assessed by global assays of coagulation.

\section{THE AIM}

In the present study, we aimed to assess the hemostatic potential of patients with liver cirrhosis and atrial fibrillation by LPTEG and to investigate changes in parameters depending on the severity of liver cirrhosis A, B, C according to ChildPugh score compared to liver cirrhosis and control.

\section{MATERIALS AND METHODS}

\section{PATIENTS AND STUDY PROCEDURE}

We performed a prospective single-center cross-sectional study at the department of internal medicine №1 of Bogomolets National Medical University, Kyiv, Ukraine, using database from departments of Internal Medicine, Gastroenterology, 
Table II. LPTEG parameters in patients with liver cirrhosis and AF, depending on the Child-Pugh score. (M $\pm \mathrm{m})$

\begin{tabular}{|c|c|c|c|c|c|}
\hline $\begin{array}{c}\text { Parameters, } \\
\text { units }\end{array}$ & $\begin{array}{l}\text { Healthy donors, } \\
\qquad n=20\end{array}$ & $\begin{array}{l}\text { Total, } \\
\mathrm{n}=70\end{array}$ & $\begin{array}{c}\text { Class A, } \\
n=25\end{array}$ & $\begin{array}{c}\text { Class } B, \\
n=31\end{array}$ & $\begin{array}{c}\text { Class C, } \\
n=14\end{array}$ \\
\hline$t_{1}, \min$. & $1,22 \pm 0,13$ & $0,7 \pm 0,03^{*}$ & $0,9 \pm 0,03$ & $0,6 \pm 0,03^{*} \#$ & $0,4 \pm 0,04^{*} \#$ \\
\hline ICC, cu. & $23,63 \pm 1,0$ & $34,58 \pm 0,77^{*}$ & $29,15 \pm 0,48$ & $35,55 \pm 0,46^{*} \#$ & $41,53 \pm 0,52 * \#$ \\
\hline CTA, cu. & $31,31 \pm 1,41$ & $47,53 \pm 0,8^{*}$ & $42,41 \pm 0,68^{*}$ & $46,6 \pm 1,02 * \#$ & $56,18 \pm 1,45^{* \#}$ \\
\hline $\mathrm{t}_{3,} \min$ & $6,72 \pm 0,28$ & $5,0 \pm 0,1^{*}$ & $5,76 \pm 0,08$ & $4,87 \pm 0,08 * \#$ & $3,9 \pm 0,14^{*} \#$ \\
\hline ICD, cu. & $34,37 \pm 1,14$ & $52,8 \pm 1,8^{*}$ & $46,06 \pm 1,06$ & $54,18 \pm 1,0 * \#$ & $73,38 \pm 1,63^{*} \#$ \\
\hline ICP, cu. & $17,08 \pm 0,45$ & $19,66 \pm 0,28^{*}$ & $17,46 \pm 0,27$ & $20,23 \pm 0,25^{*} \#$ & $22,3 \pm 0,43^{*} \#$ \\
\hline $\mathrm{t}_{5}, \min$. & $36,82 \pm 1,26$ & $32,94 \pm 0,36^{*}$ & $32,5 \pm 0,47^{*}$ & $33,56 \pm 0,33$ & $32,34 \pm 0,53^{*}$ \\
\hline MA, cu. & $536,3 \pm 11,92$ & $655,7 \pm 9,19 *$ & $589,8 \pm 9,29$ & $668,6 \pm 9,09$ & $744,8 \pm 17,29$ \\
\hline ITC, cu. & $17,91 \pm 0,66$ & $19,41 \pm 0,34^{*}$ & $18,15 \pm 0,16$ & $19,96 \pm 0,29 * \#$ & $22,94 \pm 0,55^{*} \#$ \\
\hline ICRL, \% & $0,41 \pm 0,04$ & $4,1 \pm 0,07^{*}$ & $3,73 \pm 0,13^{*}$ & $4,11 \pm 0,07^{*}$ & $4,74 \pm 0,1 * \#$ \\
\hline CTAA, cu. & $2,17 \pm 0,11$ & $2,81 \pm 0,05^{*}$ & $2,65 \pm 0,07^{*}$ & $2,68 \pm 0,05^{*}$ & $3,39 \pm 0,08^{*}$ \\
\hline
\end{tabular}

* - significance of statistical difference compared to healthy donors, $p<0,05$

\# - significance of statistical difference compared to class $A, p<0,05$

$\checkmark$ - significance of statistical difference compared to class $B, p<0,05$

Table III. LPTEG parameters in patients with liver cirrhosis, depending on the Child-Pugh score. ( $M \pm m)$

\begin{tabular}{cccccc}
\hline $\begin{array}{c}\text { Parameters, } \\
\text { units }\end{array}$ & $\begin{array}{c}\text { Healthy donors, } \\
\mathbf{n = 2 0}\end{array}$ & $\begin{array}{c}\text { Total, } \\
\mathbf{n = 3 6}\end{array}$ & $\begin{array}{c}\text { Class A, } \\
\mathbf{n = 1 3}\end{array}$ & $\begin{array}{c}\text { Class B, } \\
\mathbf{n = 1 6}\end{array}$ & $\begin{array}{c}\text { Class C, } \\
\mathbf{n = 7}\end{array}$ \\
\hline $\mathrm{t}_{1}$ min. & $1,22 \pm 0,13$ & $0,7 \pm 0,04^{*}$ & $0,9 \pm 0,05$ & $0,6 \pm 0,04^{*}$ & $0,3 \pm 0,06^{*} \#$ \\
\hline ICC, cu. & $23,63 \pm 1,0$ & $34,25 \pm 0,84^{*}$ & $29,52 \pm 0,72$ & $36,4 \pm 0,88^{*}$ & $41,29 \pm 0,97^{*} \#$ \\
\hline CTA, cu. & $31,31 \pm 1,41$ & $34,51 \pm 1,88$ & $30,29 \pm 1,48$ & $31,89 \pm 2,21$ & $48,34 \pm 5,45^{*} \# \bullet$ \\
\hline$t_{3,}$ min. & $6,72 \pm 0,28$ & $6,7 \pm 0,36$ & $7,3 \pm 0,63$ & $6,86 \pm 0,22$ & $4,54 \pm 0,26^{*} \# \bullet$ \\
\hline ICD, cu. & $34,37 \pm 1,14$ & $38,55 \pm 1,54$ & $34,95 \pm 1,35$ & $38,52 \pm 0,88$ & $53,59 \pm 1,56$ \\
\hline ICP, cu. & $17,08 \pm 0,45$ & $16,29 \pm 0,28$ & $15,22 \pm 0,43$ & $16,34 \pm 0,33$ & $18,62 \pm 0,47 \# \bullet$ \\
\hline$t_{5^{\prime}}$ min. & $36,82 \pm 1,26$ & $36,8 \pm 1,30$ & $36,8 \pm 2,24$ & $37,35 \pm 2,11$ & $35,69 \pm 1,66$ \\
\hline MA, cu. & $536,3 \pm 11,92$ & $547 \pm 19,38$ & $537,2 \pm 11,78$ & $547 \pm 34,89$ & $607,7 \pm 40,99$ \\
\hline ITC, cu. & $17,91 \pm 0,66$ & $15,09 \pm 0,56$ & $14,95 \pm 0,91$ & $14,4 \pm 0,86$ & $19,05 \pm 1,89$ \\
\hline ICRL, $\%$ & $0,41 \pm 0,04$ & $3 \pm 0,15^{*}$ & $2,34 \pm 0,11^{*}$ & $3,18 \pm 0,16^{*}$ & $3,78 \pm 0,49^{*}$ \\
\hline CTAA, cu. & $2,17 \pm 0,11$ & $2,48 \pm 0,06$ & $2,36 \pm 0,09$ & $2,38 \pm 0,07$ & $2,89 \pm 0,13^{*} \# \bullet$ \\
\hline
\end{tabular}

\footnotetext{
* - significance of statistical difference compared to healthy donors, $\mathrm{p}<0,05$

\# - significance of statistical difference compared to class $A, p<0,05$

$\checkmark$ - significance of statistical difference compared to class $B, p<0,05$
}

Hepatology and Infectious Diseases of several city hospitals. Initially we screened database between July 2020 and December 2020 for patients with determined diagnose of AF assessed by experienced cardiologists and among them we searched for patients with established diagnosis of liver cirrhosis or signs of chronic liver disease, such as elevated liver transaminases more than 3 times and bilirubin level associated with history of chronic alcohol consumption, presence of viral hepatitis serologic markers and risk factors for NAFLD. All selected patients were investigated by transient liver elastography by Soneus P7 UltraSign, Ukraine device at our department and stage of fibrosis was determined according to METAVIR score. Finally, we included 70 patients with liver cirrhosis and AF (I group), 36 patients with liver cirrhosis (II group) as comparison group and 20 healthy persons as control group. All participants were assessed and distributed according to severity of liver cirrhosis by Child-Pugh score. We excluded patients with hereditary or acquired coagulopathies of other etiologies, systemic connective tissue diseases, cancer and HIV infection. Participants who were included in this study gave their written informed consent. The study was approved by the local Ethical Committee (14.11.2019, protocol session №3) and conducted according to the Declaration of Helsinki. All patients were asked to stop taking any anticoagulants and antiagregants for 3 days before the examination. Patients baseline characteristics are described in Table I.

\section{ASSESSMENT OF LPTEG PARAMETERS}

LPTEG parameters were assessed by the portable device ARP$01 \mathrm{M}$ "Mednord". LPTEG is a global coagulation assay that measures changes of the viscoelastic properties of the blood clot 
during the process of fibrin polymerization and formation of cross-molecular bonds, its retraction and subsequent lysis. The analysis of the LPTEG graphical image is based on the changes in the relative values of the viscoelastic properties of blood (Ai) that occur during coagulation. The dynamics of the studied process - the transition of blood during coagulation from liquid to viscoelastic state - is determined and recorded as an integrated curve LPTEG, each point of which (Ai) is determined by the system at a particular time (ti). [16,17] During the study, the following LPTEG parameters were measured and analyzed:

$-t_{1}$, min - Reaction time (time in minutes from the beginning of the study to reaching the minimum amplitude of LPTG - A1). Reference values $t_{1}=0.8$ (0.6-1.3);

- Intensity of contact coagulation (ICC), conventional units (cu) - this parameter reflects mainly the aggregation activity of platelets and other blood cells, ICC reference values $=26$ (16-36);

- Constant of thrombin activity (CTA), cu - a universal criterion for estimating the intensity of the proteolytic stage of fibrin formation and thrombin generation activity. Reference values of CTA $=30$ (25-40).

$-\mathrm{t}_{3}$, min. - Blood clotting time (gel point). Reference values of $\mathrm{t}_{3}=7.4$ (5.9-9.0).

- Intensity of coagulation drive (ICD), $\mathrm{cu}$ - the resulting parameter of the intensity of the proteolytic stage of blood clotting. ICD reference values $=38(28-46)$.

- Intensity of clot polymerization (ICP), cu - characterizes the whole process of changing viscoelastic properties of the clot during polymerization of fibrin and formation of transverse intermolecular (covalent) bonds. Reference values of ICP $=18.5(15.4-22.5)$ for 10 minutes.

- Maximum amplitude (MA), cu - describes the maximum clot density due to platelet activity, and quantitative / qualitative characteristics of fibrin polymerization. Reference values of MA $=510(450-650)$

$-t_{5}$, min. - time to reaching the maximum amplitude of LPTEG. Reference values of $t_{5}=30.7$ (23.3-39).

- Intensity of total coagulation (ITC), $\mathrm{cu}$ - characterizes the general trend of coagulation due to the platelet aggregation capacity, activity of the proteolytic stage of coagulation and maximum clot amplitude. Reference values of ITC $=17.04$ (14.68-20.12).

- Intensity of clot retraction and lysis (ICRL), \%. - describes the activity of the fibrinolytic system. Reference values of ICLR $=0.29(0.27-2.3) \%$.

- Coefficient of total anticoagulant activity (CTAA), $\mathrm{cu}$ - total anticoagulant activity of blood due to serine protease inhibitors, coenzyme inhibitors (proteins C, S, thrombomodulin), inhibitors of active complexes (TFPI), Reference values of CTAA $=2,2(1,8-2,5)$.

Blood sampling was performed from a vein with a $1.0 \mathrm{ml}$ three-component silicone syringe with a rubber cuff. The obtained blood sample was immediately placed in a 0.45 $\mathrm{ml}$ cuvette during 30 seconds and the study was started.

\section{STATISTICAL ANALYSIS}

Statistical analysis was performed using the Excel for Windows and IBM SPSS Statistics software package.
Methods of parametric and nonparametric statistics were used. The distribution of quantitative values was assessed using the Kolmogorov - Smirnov criterion. Student's t-test was used to compare the mean values of the parameters for 2 independent samples with normal distribution, and Mann-Whitney U test for not normally distributed values. The nonparametric Kruskal-Wallis test was used for multiple comparisons.

\section{RESULTS}

Parameters of LPTEG were obtained and analyzed in all patients with liver cirrhosis and $\mathrm{AF}$ depending on the severity of cirrhosis according to Child-Pugh score and compared to similar parameters of patients with liver cirrhosis and healthy individuals. (Table II. and Table III).

Reaction time $\left(t_{1}\right)$ was significantly reduced compared to control, and ICC parameter was substantially increased $(\mathrm{p}<0.001)$. There was a significant reduction in $\mathrm{t}_{1}$ time and an increase in ICC between controls and Child-Pugh classes $\mathrm{B}, \mathrm{C}, \mathrm{p}<0.01$, as well as a gradual reduction in $\mathrm{t}_{1}$ and an increase in ICC with worsening liver cirrhosis. The mean values of $t_{1}$ and ICC between 2 groups were at the same level and did not differ statistically ( $\mathrm{p}>0.05)$. The mean levels of CTA in patients with liver cirrhosis and AF were higher than in control group and group of liver cirrhosis $(p<0.001)$. A significant difference was found between healthy individuals and patients of classes A, B, C by ChildPugh score $(p<0.01)$, with a gradual increase in CTA as the severity of liver dysfunction progresses. The mean Blood clotting time $\left(\mathrm{t}_{3}\right)$ was significantly reduced compared to healthy individuals and liver cirrhosis group $(p<0.001) . T_{3}$ of class A did not differ from the $t_{3}$ of normal individuals $(p=0.823)$ and gradually decreased with increasing severity of liver cirrhosis, $\mathrm{p}<0.01$. ICD was significantly increased compared to control $(\mathrm{p}<0.001)$ and compared to liver cirrhosis $(p=0.001)$. The mean level of ICD had a tendency to increase with worsening liver dysfunction $(p<0.05)$. ICP parameter was substantially increased compared to healthy individuals and liver cirrhosis $(\mathrm{p}<0.001)$ The ICP gradually increased, gaining a statistically significant difference between control and classes $\mathrm{B}, \mathrm{C}, \mathrm{p}<0.01$ and between classes $\mathrm{A}$ and $\mathrm{C}$ according to Child-Pugh score, $\mathrm{p}<0.01$. MA was significantly increased compared to control and liver cirrhosis $(p<0.001)$ There was a tendency to increasing of MA from class A to $\mathrm{C}, \mathrm{p}<0.01 \mathrm{~T}_{5}$ time was slightly reduced compared to control and liver cirrhosis group $(\mathrm{p}<0.01)$. $\mathrm{t}_{5}$ differed statistically only between control and Child-Pugh classes A, C ( $<<0.01)$. The general coagulation trend ITC was increased compared to healthy individuals $(\mathrm{p}<0.01)$ and liver cirrhosis $(\mathrm{p}<0.001)$ and exceeded the reference values, increasing with the progression of the severity of liver cirrhosis, indicating a state of hypercoagulation $(\mathrm{p}<0.01)$. ICRL was significantly increased compared to control and liver cirrhosis $(\mathrm{p}<0.001)$ Fibrinolysis activity increased with increasing severity of liver cirrhosis, obtaining a statistical difference between Child-Pugh classes A and $C, p<0.01$. CTAA was significantly increased compared 
to healthy individuals and liver cirrhosis $(\mathrm{p}<0.001)$. There was a substantial statistical difference between the control and Child-Pugh classes A, B, C ( $\mathrm{p}<0.01)$, a slight difference between classes $B$ and $C(p=0.49)$.

\section{DISCUSSION}

In this prospective cross-sectional study of patients with liver cirrhosis and atrial fibrillation we investigated LPTEG parameters and found out that all patients had obvious coagulopathy abnormalities at primary, secondary and fibrinolytic stages of hemostasis compared to healthy donors and liver cirrhosis. Particularly, at the primary stage we observed increased aggregation of platelets and decreased rheological blood stability. As parameters of aggregation activity $t_{1}$ and ICC were almost the same in patients of 2 groups, we made a conclusion that AF has no effect on platelet aggregation activity and such suggestion explains the low effectiveness of antiplatelet drugs for prevention of thrombotic complications in patients with AF. $[18,19]$

Significant activation of secondary stage of coagulation and thrombin generation was observed due to an increase in the CTA parameter. The intensity of coagulation is largely determined by the activity of thrombin, which is the main enzyme activator of the whole coagulation process, as it participates in the conversion of fibrinogen to fibrin, activates platelets, coagulation factors V, VIII, XIII, tissue plasminogen factor, and, furthermore, participates in systemic inflammation.[20] In patients with liver cirrhosis and AF intensive thrombin generation was observed at all stages according to Child-Pugh, the cause of which is currently unknown. However, AF alone may be associated with decreased ability to activate protein $\mathrm{C}$ due to decreased thrombomodulin expression on the background of structural changes in the left atrium. [21] Hyperfibrinolysis in patients with liver cirrhosis can be explained by the reduced synthesis of thrombomodulin and reduced activity of fibrinolysis inhibitors (TAFI), which inhibit the conversion of plasminogen to plasmin and is further exacerbated by atrial fibrillation. [22]

The existing evidence on coagulation process in patients with liver cirrhosis and AF is quite limited and we made first steps in understanding these complex hemostasis alterations.

Our study has limitations. The mean age of patients with liver cirrhosis and AF is not completely comparable with the age of patients with liver cirrhosis, because on average the I group of patients is older than II by 10 years $(68.3 \pm 1.08$ vs. $58.3 \pm 1.45)$. Also, in our study, we did not investigate differences in hemostatic potential depending on gender, age, etiology of liver cirrhosis. Therefore, we assume that these factors may have influenced the result.

\section{CONCLUSIONS}

Patients with liver cirrhosis have a normal hemostatic potential with a slight shift towards hypercoagulation at the stage of decompensation. When associated with AF, hemostatic potential shifts significantly in the direction of hypercoagulation with a gradual worsening of coagulation disorders, starting from the compensated stage of cirrhosis severity. We showed that global coagulation assays can more precisely describe complex alterations in hemostasis in patients with liver cirrhosis and AF and should be commonly implemented in routine clinical practice along with conventional coagulation parameters. Some LPTEG parameters as CTA can be a promising biomarkers for identifying individuals with increased thrombotic risk and anticoagulant treatment should be considered as a benefit option for this population

\section{REFERENCES}

1. Roth G.A., Abate D., Abate K.H. et al. Global, regional, and national age-sex-specific mortality for 282 causes of death in 195 countries and territories, 1980-2017: a systematic analysis for the Global Burden of Disease Study 2017.Lancet. 2018;392(10159):1736-1788

2. Asrani S.K., Devarbhavi H. Burden of liver diseases in the world. J Hepatol. 2019; 70(1):151-171.

3. Sarin S.K., Maiwall R. Global Burden Of Liver Disease: A True Burden on Health Sciences and Economies. World Gastroenterology Organisation. https://www.worldgastroenterology.org/publications/e-wgn/e-wgnexpert-point-of-view-articles-collection/global-burden-of-liverdisease-a-true-burden-on-health-sciences-and-economies.

4. Munger T.M., Wu L.Q., Shen W.K. Atrial Fibrillation. JBR. 2014;28(1):1-17.

5. Chung M.K., Eckhardt L.L., Chen L.Y. et al. Lifestyle and Risk Factor Modification for Reduction of Atrial Fibrillation: A Scientific Statement from the American Heart Association. Circulation. 2020; 141 (16):750-772.

6. Darrat Y.H., Smer A., Elayi C.S. et al. Mortality and morbidity in patients with atrial fibrillation and liver cirrhosis; World J Cardiol. 2020; 12(7): 342-350.

7. Chokesuwattanaskul R., Thongprayoon C., Bathini T. Epidemiology of atrial fibrillation in patients with cirrhosis and clinical significance: a meta-analysis. Eur J Gastroenterol Hepatol. 2019;31(4):514-519.

8. Khoury T., Ayman A.R., Cohen J.etal. The Complex Role of Anticoagulation in Cirrhosis: An Updated Review of Where We Are and Where We Are Going. Digestion. 2016;93:149-159.

9. Zermatten M.G., Fraga M., Moradpour D. et al. Haemostatic alterations in cirrhotic patients: from primary haemostasis to fibrinolysis. Hepatology. 2020; 71:2135-2148.

10. Forkin K., Colquhoun D., Huffmyer J. The Coagulation Profile of EndStage Liver Disease and Considerations for Intraoperative Management. Anesthesia \& Analgesia.2018;126 (1):46-61.

11. Harrison M.F. The Misunderstood Coagulopathy of Liver Disease: A Review for the Acute Setting. West J Emerg Med. 2018;19(5): 863-871.

12. Kaski J.C., Arrebola-Moreno A.L. Inflammation and Thrombosis in Atrial Fibrillation. Rev. Esp. Cardiol. 2011;64(7):551-553.

13. Benes J., Zatloukal J., Kletecka J. Viscoelastic Methods of Blood Clotting Assessment. A Multidisciplinary Review. Front Med (Lausanne). 2015;2:62.

14. Hans G.A., Besser M.W. The place of viscoelastic testing in clinical practice. BJHaem. 2016;173(1):37-48.

15. Shen L., Tabaie S., Ivascu N. Viscoelastic testing inside and beyond the operating room. J Thorac Dis. 2017;9(4):299-308.

16. Tutrin I., Udut V. Nizkochastotnaya pyezotromboelastografiya tselnoy krovi: algoritmy diagnostiki i korrektsii gemostaziologicheskikh rasstroystv. [Low-frequency piezoelectric thromboelastography of whole blood: algorithms for diagnostics and correction of hemostasis disorders]. Tomsk: Publishing House of Tomsk State University. 2016:1358. (In Russian). 
17. Tarabrin 0., Shcherbakov S., Gavrychenko D. et al. Low-Frequency Piezoelectric Thromboelastography Vs Platelet Aggregation Test, Standard Coagulation Tests and Thromboelastography. Anesthesia \& Analgesia. 2016;123(3):173.

18. Sardana M., Hwang S.J., Puurunen M.K. et al. Association of platelet aggregation with atrial fibrillation: The Framingham Offspring Study. J Am Coll Cardiol. 2018;71 (11).

19. Manaktala R., Kluger J. Role of Antiplatelet Therapy in Stroke Prevention in Patients with Atrial Fibrillation. J Am Osteopath Assoc. 2017; 117(12):761-771.

20. Crawley J., Zanardelli S., Chion A. The central role of thrombin in hemostasis. Journal of Thrombosis and Haemostasis 2007;1(1):95-101.

21. Cervero J., Montes R., Espana F. et al. Limited ability to activate protein C confers left atrial endocardium a thrombogenic phenotype. A role in cardioembolic stroke? Stroke. 2011;42(9): 10.1161/ STROKEAHA.111.614420.

22. Colucci M., Binetti B.M., Branca M.G. et al. Deficiency of thrombin activatable fibrinolysis inhibitor in cirrhosis is associated with increased plasma fibrinolysis. Hepatology.2003;38:230-237.

This research work is a part of Department Scientific Research work "Optimization of diagnosis and treatment of diseases of the digestive system in conditions of comorbidity" (2019-2022, №0119U100576). This work didn't receive any grants or other financial sources.

\section{ORCID and contributionship:}

Alina E. Baylo: 0000-0003-3519-9788 A, B, C, D

Tetiana O. Maksymets: 0000-0003-4530-7543 ${ }^{B, E}$

Vadim P. Shypulin: 0000-0002-6780-130X ${ }^{F}$

Volodymyr V. Cherniavskyi: 0000-0001-5831-8810 ${ }^{\text {B, E }}$

Luiza M. Parunian: 0000-0002-8370-2045 ${ }^{B}$

\section{Conflict of interest:}

The Authors declare no conflict of interest.

\section{CORRESPONDING AUTHOR Alina E. Baylo \\ Bogomolets National Medical University 13 Taras Shevchenko Boulevard, 01601 Kyiv, Ukraine tel: +380996503957 \\ e-mail:alinabajlo@gmail.com}

Received: 04.10 .2020

Accepted: 02.03 .2021

A - Work concept and design, B - Data collection and analysis, C - Responsibility for statistical analysis, D-Writing the article, $\mathbf{E}$-Critical review, $\mathbf{F}$ - Final approval of the article 